\title{
BMJ Open Implementation of blood glucose self- monitoring among insulin-dependent patients with type 2 diabetes in three rural districts in Rwanda: 6 months open randomised controlled trial
}

\author{
Loise Ng'ang'a (D) , ${ }^{1}$ Gedeon Ngoga, ${ }^{2,3}$ Symaque Dusabeyezu, ${ }^{1}$ \\ Bethany L Hedt-Gauthier, ${ }^{4}$ Patient Ngamije, ${ }^{5}$ Michel Habiyaremye, ${ }^{6}$ \\ Emmanuel Harerimana, ${ }^{1}$ Gilles Ndayisaba, ${ }^{2}$ Christian Rusangwa, ${ }^{1}$ \\ Simon Pierre Niyonsenga, ${ }^{2}$ Charlotte M Bavuma, ${ }^{7}$ Gene Bukhman, ${ }^{3,4,8}$ \\ Alma J Adler, ${ }^{4}$ Fredrick Kateera, ${ }^{1}$ Paul H. Park ${ }^{3,4,8}$
}

To cite: Ng'ang'a L, Ngoga G, Dusabeyezu S, et al. Implementation of blood glucose self-monitoring among insulin-dependent patients with type 2 diabetes in three rural districts in Rwanda: 6 months open randomised controlled trial. BMJ Open 2020;10:e036202. doi:10.1136/ bmjopen-2019-036202

- Prepublication history for this paper is available online. To view these files, please visit the journal online (http://dx.doi. org/10.1136/bmjopen-2019036202).

Received 11 December 2019 Revised 07 April 2020 Accepted 17 June 2020

Check for updates

(c) Author(s) (or their employer(s)) 2020. Re-use permitted under CC BY-NC. No commercial re-use. See rights and permissions. Published by BMJ.

For numbered affiliations see end of article.

Correspondence to

Loise Ng'ang'a;

loisehaks@gmail.com

\section{ABSTRACT}

Introduction Most patients diagnosed with diabetes in sub-Saharan Africa (SSA) present with poorly controlled blood glucose, which is associated with increased risks of complications and greater financial burden on both the patients and health systems. Insulin-dependent patients with diabetes in SSA lack appropriate home-based monitoring technology to inform themselves and clinicians of the daily fluctuations in blood glucose. Without sufficient home-based data, insulin adjustments are not data driven and adopting individual behavioural change for glucose control in SSA does not have a systematic path towards improvement.

Methods and analysis This study explores the feasibility and impact of implementing self-monitoring of blood glucose (SMBG) in patients with type 2 diabetes in rural Rwandan districts. This is an open randomised controlled trial comprising of two arms: (1) Intervention groupparticipants will receive a glucose metre, blood test strips, logbook, waste management box and training on how to conduct SMBG in additional to usual care and (2) Control group - participants will receive usual care, comprising of clinical consultations and routine monthly follow-up. We will conduct qualitative interviews at enrolment and at the end of the study to assess knowledge of diabetes. At the end of the study period, we will interview clinicians and participants to assess the perceived usefulness, facilitators and barriers of SMBG. The primary outcomes are change in haemoglobin A1c, fidelity to SMBG protocol by patients, appropriateness and adverse effects resulting from SMBG. Secondary outcomes include reliability and acceptability of SMBG and change in the quality of life of the participants. Ethics and dissemination This study has been approved by the Rwanda National Ethics Committee (Kigali, Rwanda No.102/RNEC/2018). We will disseminate the findings of this study through presentations within our study settings, scientific conferences and publication in a peer-reviewed journal.

Trial registration number PACTR201905538846394; pre-results.
Strengths and limitations of this study

- To our knowledge, this is the first open randomised trial to study the feasibility of self-monitoring of blood glucose (SMBG) in rural sub-Saharan Africa.

- Qualitative interviews will be conducted to provide patients and providers perspectives on developing a contextually appropriate SMBG.

- Results do not reflect long-term feasibility of SMBG.

\section{INTRODUCTION}

The endemic burden of diabetes in subSaharan Africa (SSA) is of growing global health concern. It is characterised by inadequate data, weak infrastructure, limited diagnostic and treatment capacity, and limited human resources leading to disproportionate disability and mortality among the rural and poor populations. ${ }^{1-3}$ Furthermore, impoverished areas are more likely to include atypical forms of diabetes among malnourished children and young adults. ${ }^{4}$ The prevalence of diabetes in SSA is often under-reported, with approximately two-thirds of those with diabetes undiagnosed. ${ }^{5}$ While there is an unclear pathophysiology and burden of diabetes in much of SSA, the enormous social and financial hardship for patients due to the disease's chronic nature and health complications is well documented. ${ }^{67}$ To avoid such hardships, good glycaemic control is considered the primary target towards preventing organ failure and other diabetes complication; however, the majority of patients with diabetes in SSA have uncontrolled blood glucose levels. ${ }^{8}$ Given the poorly controlled 
diabetes in SSA, it is urgent to explore new, innovative means of care delivery as to address this shortcoming among an already vulnerable population.

Tracking of blood glucose levels by patients and care providers remains an integral component in the management of a diabetes patient. ${ }^{9-11}$ Evidence, primarily from high-income countries, has illustrated the effectiveness of self-monitoring of blood glucose (SMBG) on controlling diabetes. ${ }^{12}{ }^{13}$ However, there are limited data on the feasibility and impact of SMBG among patients in the rural regions of SSA. The few available studies in resourcelimited settings have shown that SMBG is impeded by several contextual challenges. First, there is limited awareness of and access to SMBG for patients, and costs of glucose metres and test strips are prohibitive. ${ }^{14-16}$ Among the few patients who have access to SMBG, performance of blood glucose measurements is irregular, with poor record keeping ${ }^{16}$ and suboptimal adherence to the prescribed SMBG schedule. As a result, the majority of insulin-dependent patients in these settings are not able to effectively monitor and correct hypoglycaemic and hyperglycaemic events. Collectively, these access and feasibility challenges lead to a lack of data to inform decisions on optimal diabetes care, including but not limited to informing data-driven insulin dose adjustments. ${ }^{6}{ }^{17}$ As a result, clinicians oftentimes only rely on a single random blood sugar reading obtained during the routine patient visits. Without knowledge of patients' blood glucose levels at different intervals of the day, healthcare providers may underdose insulin to avoid hypoglycaemic events. To collect glucose data at home and guide clinicians to make more accurate insulin dose adjustments and customised health education, there is an urgent need to design efforts aimed at optimising the implementation of SMBG for all insulin-dependent patients within these settings. In this study, we will prospectively assess the feasibility and impact of implementing an innovative, community-based SMBG approach among patients with insulin-dependent type 2 diabetes (T2D) managed in three district hospital noncommunicable disease (NCD) clinics in rural Rwanda.

\section{Study objectives and aims}

With this implementation research project, we will monitor and evaluate the feasibility and impact of the SMBG on glycaemic control. Outlined below are the specific objectives:

\section{Aim 1}

To assess the feasibility of performing SMBG among insulin dependent T2D patients, perceived barriers, facilitators and usefulness among NCD clinicians in rural Rwanda.

\section{Aim 2}

Evaluate SMBG's early impact on glycaemic control and diabetes knowledge change among the patients with insulin-dependent T2D.

\section{METHODS AND ANALYSIS}

\section{Setting}

This open randomised trial will be conducted in three rural Rwandan district hospitals-Kirehe and Rwinkwavu district hospitals located in Eastern province and Butaro district hospital located in Northern province of Rwanda. The hospitals are run by the Rwandan Ministry of Health $(\mathrm{MOH})$ and supported by Partners In Health (PIH)/ Inshuti Mu Buzima (IMB), a non-governmental organisation that has been supporting the country's initiative to strengthen rural health services since 2005. By 2009, the $\mathrm{MOH}$ and IMB implemented nurse-led integrated NCD clinics within each of the three district hospitals that serve a combined catchment population of over 800000 individuals. ${ }^{18}$ This integrated NCD clinic model is well described elsewhere. ${ }^{19-25}$ Briefly, the NCD nurses receive specialised training on the integrated management of severe, chronic NCDs, including type 1 diabetes and insulin-dependent T2D, heart failure, severe hypertension and severe asthma. The nurses also receive monthly one-on-one supervision and mentoring provided by an endocrinologist and a cardiologist. Each of the district hospitals hosts diabetes clinic once (Rwinkwavu and Butaro) or twice (Kirehe) per week. All patients seen at these clinics are scheduled for follow-up consultations appointments at least once a month.

Management of patients in these clinics is guided by standardised national diabetes treatment protocols, developed by the Rwandan $\mathrm{MOH}$ in collaboration with PIH/IMB and other partners. Patients with T2D who are in need of insulin are admitted to the district hospital internal medicine wards for insulin initiation and to allow for patient and family education on insulin management. ${ }^{21}$ Patients then receive chronic care through the outpatient NCD clinics with routine monitoring for complications and laboratory tests, including haemoglobin A1c (HbA1c) and creatinine.

\section{Study design}

This is an open randomised trial to assess the feasibility and impact of SMBG among patients with insulindependent T2D in three rural districts in Rwanda.

\section{Open randomised control trial}

We will conduct an open randomised trial consisting of two study arms: Arm (1) In the

intervention group-we will provide participants with a glucose metre (SD Code Free 01GM11), blood glucose test strips, logbook, a waste management box and training on how to conduct SMBG. The training will be divided into two sessions, the first session will be conducted as a group session where enrolled participants will be trained on the process of performing effective SMBG and signs and symptoms of hyper- and hypoglycaemia. Each participant will be required to demonstrate the process of SMBG including recording the glucose readings into the logbook. The training session will take approximately 4 
hours and will be facilitated by qualified study staff with support from NCD clinicians.

Arm (2) In the control arm, participants will continue to receive usual diabetes care. This will include routine monthly follow-up for consultation with random blood glucose check, usual insulin prescription and measurement of HbAlc at baseline, three months and at six months.

\section{Qualitative interviews}

We will use semistructured qualitative interview research methods to (1) identify the preintervention and postintervention knowledge of diabetes among the sample population (2) identify the facilitators and barriers to performing SMBG among the participants and (3) explore the perceived usefulness of SMBG among NCD clinicians in rural Rwanda. We will use purposive sampling by age, duration of diabetes, sex and type of diabetes to identify participants of the semistructured qualitative interviews. We have developed a facilitator guide that has been translated to local Kinyarwanda language. Probing techniques will be used to prompt more responses from the participants. All the qualitative interview sessions will be facilitated by qualified and trained study staff. The interviews will be audio recorded, transcribed and translated from Kinyarwanda to English.

\section{Randomisation}

Participants will be individually randomised using a random number table. Allocation will be concealed using sealed envelopes. One person will be responsible for the randomisation process at all sites and this person will not have access to the patient clinical records. Each site will have an equal number of participants allocated to the intervention and control arm.

\section{Study population and sample size}

All patients with type 2 diabetes who are managed using insulin at any of the three $\mathrm{MOH} / \mathrm{PIH}$ supported district hospitals will be eligible to participate in this study.

The power of this study was calculated based on the primary outcome of interest, change in HbA1c. Based on existing literature, we anticipate a $0.2 \%$ change in HbAlc among the control ${ }^{19}$ group and a $1.2 \%$ change among participants enrolled in the intervention group. ${ }^{26}$ The total number of participants enrolled in this study is 82 ( 45 for interventions and 37 for controls). Figure 1 presents power for a range of assumed SD; for SD of 1.9 or less, the study has greater than $80 \%$ power to detect the mean difference of the change in HbAlc among the intervention compared with control group.

\section{Inclusion and exclusion criteria}

The inclusion criteria comprises of adult patients at least 18 years of age, a diagnosis of T2D, on insulin regimen at the time of study enrollment, either possess the ability to read and write sufficiently to use logbooks or have a reliable access to a guardian that can read and write, and have HbAlc reading of $7 \%$ or above. Exclusion criteria

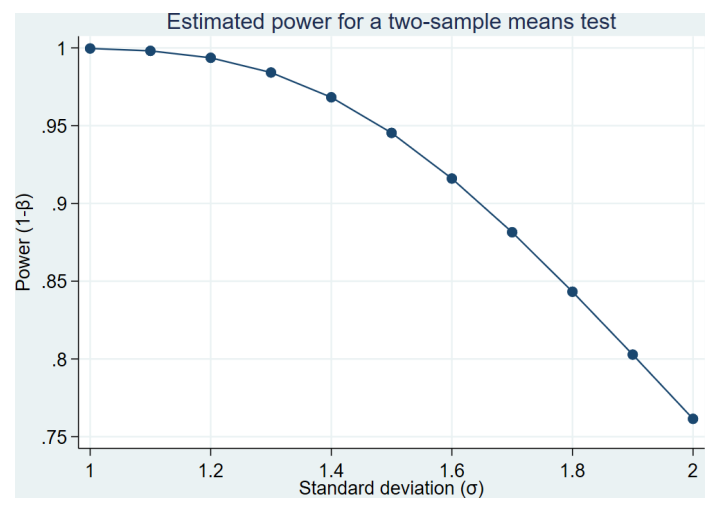

Figure 1 Caption-estimated power for a two sample means test to detect the mean difference of the 6 months change in $\mathrm{HbA1c}$ among the interventions group who are implementing self-monitoring of blood glucose in addition to usual type 2 diabetes care compared with the control group who are only receiving usual care in the management of type 2 diabetes. Parameters used: $\alpha=0.05 \mu_{1}=0.2, \mu_{2}=1.2$. Sample size $(N)=82$; $\mathrm{N} 1$ (control)=37, N2 (intervention) $=45$. HbA1c, haemoglobin A1c.

comprises of a diagnosis of type 1 diabetes, gestational diabetes, chronic kidney disease (elevated creatinine levels above $200 \mu \mathrm{mol} / \mathrm{L}$ ), inability to read and write and have no access to a reliable guardian who can read and write sufficiently to use logbooks.

\section{Data collection}

Enrolment for this study has already occurred between April and June 2019. Patients will be followed for 6 months after the date of participant enrollment; Final follow-up data will be collected in December 2019 and the final end-line qualitative studies will be conducted in February 2020. A second phase of data collection, involving chart review will be conducted between April and June 2020. The data collection process is facilitated by three trained study staff. Study data will be collected as follows (1) a complete intake form-to confirm the eligibility to the study (2) a brief survey on demographic and basic clinical characteristics (3) a WHO quality of life questionnaire (4) a HbAlc test (5) qualitative interviews conducted on 12 of the study participants and (6) chart review to obtain information on treatment dosages and adjustments among the study participants

\section{Participant selection process}

Eligible study participants were identified through the electronic medical records. The list of participants was extracted separately for each district hospital. We also conducted chart review and consulted the NCD clinicians to identify eligible participants. The study staff then contacted these participants during routine follow-up to obtain an informed consent for enrolment into the study.

\section{Intervention group}

In addition to the data collection above, on enrolment, the participants of the intervention group were given instructions and training on the following topics: (1) 
appropriate use of glucose metre machines, test strips, and blood lancets, including avoiding cross utilisation by other members, (2) proper waste disposal mechanisms, (3) signs and symptoms of hypo and hyperglycaemia, and necessary action to follow in case of hypo or hyperglycaemic episodes, (4) Schedule and frequency of blood glucose testing; participants will be expected to measure their premeal blood sugar levels 7 days a week, performing one test every day. The daily tests will be alternated to perform either morning, midday or evening tests on predefined days of the week. This schedule/ frequency will provide a daily blood sugar reading among the participants. Due to the cost of test strips and being the initial time SMBG is being introduced within this study population, we are assuming that seven readings a week, conducted at different times of the day will provide significant information to guide the providers' decision-making process of insulin titration, customised health education and an understanding towards developing a culturally and contextually appropriate SMBG programme for patients with diabetes in rural Rwanda. The participants have also been given a mobile phone number that they can call or text to reach a study staff in case of any questions or concerns related to SMBG.

In addition, patients in this group will be required to carry their glucose metre machines and logbooks during their usual routine clinic follow-up scheduled on a monthly basis. During these visits, the care providers will assess the recorded glucose information to inform management and the study staff will obtain data on both the utilisation of the logbook and the glucose metre machine.

\section{Study outcomes}

The primary and secondary outcomes of this study are described as follows

\section{- Primary outcomes:}

Change in HbAlc: This assay will be performed at the study setting using a point-of-care device and requires a lancet-induced drop of blood from the participant's fingertip. The resulting per cent value reflects the blood glucose level over the past 1-3 months. This will be measured at study enrolment, 3 months after enrolment and on conclusion of the study period.

Fidelity: Several variables will reflect the participants' adherence to the utilisation of technology as described in the training. These include use of glucose metre and log book. We will assess the proportion of number of times the blood sugar was checked and recorded compared with the expected number of blood sugar tests as prescribed. We will assess predictors of patient's adherence to SMBG.

Appropriateness: Several factors will be assessed from both quantitative and qualitative data. The participants will be asked questions in qualitative interviews surrounding the ease of use and benefits of such technology in their setting.
Severe adverse events: Potential adverse events include infection, local skin reaction, bleeding, hospitalisation, hypoglycaemia and hyperglycaemia. Data sources will include self-reports in logbooks, reporting by clinicians and qualitative interviews.

- Secondary:

Vital status: proportion of participants who die,-number of persons lost to follow-up, number of participants alive and in-care will be assessed.

Acceptability: Participants and clinical providers will be interviewed on their satisfaction with SMBG technologies, specifically around its content, complexity, comfort, delivery and costs they would be willing to pay to sustain SMBG.

Reliability of logbooks: This will be measured in the intervention arm by comparing what is recorded in the logbook to what is automatically recorded by the glucose metre machine.

Quality of life: WHO quality of life surveys will be conducted at the start and conclusion of the study period.

Treatment adjustment: The participants' diabetes treatment regimen will be collected at study enrolment. Changes to the treatment regimen will be tracked and assessed following the routine monthly clinic visits among the study participants. This information will be collected through chart review of the study participants' medical records.

\section{Data analysis plan}

For quantitative outcomes, descriptive statistics will be used. Specifically, to assess the first aim on the feasibility of SMBG, we will report frequencies, proportions and percentages. We will use a paired t-test to assess the change in HbAlc within the study period. We will also conduct analysis to compare the mean difference of the HbAlc change between the intervention and control groups. To control for other factors that may be associated with the change in HbA1C, we will perform a linear regression, where the outcome variable is the change in HbAlc while the other factors are controlled. To test the association of patients' characteristics and 'adherence to SMBG', we will conduct bivariate and multivariate regression analysis. We will use difference in difference technique to assess the quality of life among the study participants before and after implementation of SMBG. To minimise factors that may be caused by differences in the three district hospitals, we will control for health facility site in our analysis.

For qualitative analysis, interviews will be transcribed then translated from Kinyarwanda to English. We will use MAXQDA to conduct content analysis. Specific quotations, phrases or group interactions will be used to develop codes. These codes will then be grouped together to develop distinct themes. Deductive analysis will be used to identify priori themes while inductive analysis will be used to identify emerging codes. Note that deductive analysis will be used to identify a priori themes including; (1) perception of using home glucometer and logbook 
(2) perception of testing frequency (3) perception of using logbook to inform patient management. Inductive analysis will also be used to identify other significant codes and themes. The quantitative and qualitative data analysis will be conducted independently.

\section{Patients and public research involvement}

We will engage the patients throughout the study period. As a feasibility study, we will seek the perspectives and views of implementing SMBG among the patients recruited for this study. This includes seeking their opinions on the perceived usefulness, benefits, barriers and cues to actions towards developing an effective SMBG programme and future SMBG research activities appropriate for implementation within the rural districts of Rwanda.

\section{Ethics and dissemination plan}

The protocol has been approved by the Rwanda National Ethics Committee (Kigali, Rwanda No. 102/RNEC/2018). Each participant will be required to provide a written informed consent prior to enrolment in the study. We will disseminate these study findings to patients through oral presentations conducted during health education sessions. We will also disseminate the findings to the clinicians and hospital leadership within our study catchment area . In addition, we will present the findings in local and international scientific conferences as well as publish manuscripts peer-reviewed journals.

\section{Discussion}

The global burden of diabetes and other NCDs is of substantial concern with disproportionate impacts in rural SSA. Insulin-dependent diabetes in particular requires a new care delivery design to reverse the current trend of poorly controlled disease, which leads to lethal complications and further socioeconomic burden. To our knowledge, this is the first open trial assessing the impact and feasibility of implementing SMBG in rural SSA. Adherence to SMBG protocols by patients as well as clinical outcomes, such as HbAlc, will inform the feasibility and effectiveness of SMBG in a rural SSA population. Our study findings aim to demonstrate important aspects in developing a culturally and contextually appropriate SMBG programme for patients with diabetes mellitus in rural Rwanda and other rural areas of SSA at large. It also aims at assessing the added value of SMBG in achieving glycaemic control targets and improving the quality of life for patients with diabetes. The outcomes of the study will serve as advocacy for greater access and further studies on SMBG especially among patients residing in rural SSA.

\footnotetext{
Author affiliations

${ }^{1}$ Research, Inshuti Mu Buzima, Partners In Health-Rwanda, Rwinkwavu, Rwanda ${ }^{2}$ Non-Communicable Diseases Division, Rwanda Biomedical Center, Kigali, Rwanda ${ }^{3}$ NCD Synergies, Partners in Health, Boston, Massachusetts, United States

${ }^{4}$ Global Health and Social Medicine, Harvard Medical School, Boston, Massachusetts, USA

${ }^{5}$ Kirehe District Hospital, Ministry of Health, Kigali, Rwanda

${ }^{6}$ Rwinkwavu District Hospital, Ministry of Health, Kigali, Rwanda
}

${ }^{7}$ Internal Medicine, University of Rwanda College of Medicine and Health Sciences, Kigali, Rwanda

${ }^{8}$ Division of Global Health Equity, Brigham and Women's Hospital, Boston, Massachusetts, USA

Acknowledgements The authors would like to thank the SMBG research team led by Samuel Haragirimana and the NCD coordinators for their assistance with all aspects of this study. We thank Partners In Health, Rwanda for providing staff time and data collection support towards this study. We also thank the clinicians who are involved in the management of type 2 diabetes patients involved in this study. Finally, we thank all the type 2 diabetes patients who are involved in this study.

Contributors PP and GN are the principal investigators of this study protocol. LN led the literature review and drafting of the initial draft of the protocol and the manuscript draft. AJA contributed to the overall protocol development and study design. SD, BLH-G, PN, MH, EH, GN, CR, SPN, CMB, GB and FK contributed to the overall protocol development. All authors reviewed and commented on the final draft of this protocol manuscript

Funding The authors have not declared a specific grant for this research from any funding agency in the public, commercial or not-for-profit sectors.

Competing interests None declared.

Patient and public involvement Patients and/or the public were involved in the design, or conduct, or reporting, or dissemination plans of this research. Refer to the Methods section for further details.

Patient consent for publication Not required.

Provenance and peer review Not commissioned; externally peer reviewed.

Open access This is an open access article distributed in accordance with the Creative Commons Attribution Non Commercial (CC BY-NC 4.0) license, which permits others to distribute, remix, adapt, build upon this work non-commercially, and license their derivative works on different terms, provided the original work is properly cited, appropriate credit is given, any changes made indicated, and the use is non-commercial. See: http://creativecommons.org/licenses/by-nc/4.0/.

ORCID iD

Loise Ng'ang'a http://orcid.org/0000-0002-1045-0780

\section{REFERENCES}

1 Bukhman G, Bavuma C, Gishoma C, et al. Endemic diabetes in the world's poorest people. Lancet Diabetes Endocrinol 2015;3:402-3.

2 Strasser R, Kam SM, Regalado SM. Rural health care access and policy in developing countries. Annu Rev Public Health 2016;37:395-412.

3 Hall V, Thomsen RW, Henriksen O, et al. Diabetes in sub Saharan Africa 1999-2011: epidemiology and public health implications. A systematic review. BMC Public Health 2011;11:564.

4 Alemu S, Dessie A, Seid E, et al. Insulin-Requiring diabetes in rural Ethiopia: should we reopen the case for malnutrition-related diabetes? Diabetologia 2009;52:1842-5.

5 Mbanya J-C, Motala AA, Sobngwi E, et al. Review of the prevalence of diabetes mellitus in sub-Saharan Africa. Lancet 2010;375:2254-66.

6 Nuche-Berenguer B, Kupfer LE. Erratum to "readiness of subSaharan Africa healthcare systems for the new pandemic, diabetes: a systematic review". J Diabetes Res 2018;2018:1-2.

7 Anyangwe SCE, Mtonga C. Inequities in the global health workforce: the greatest impediment to health in sub-Saharan Africa. Int $J$ Environ Res Public Health 2007;4:93-100.

8 Camara A, Baldé NM, Sobngwi-Tambekou J, et al. Poor glycemic control in type 2 diabetes in the South of the Sahara: the issue of limited access to an HbA1c test. Diabetes Res Clin Pract 2015;108:187-92.

9 Goldstein DE, Little RR, Lorenz RA, et al. Tests of glycemia in diabetes. Diabetes Care 2004;27:1761-73.

10 Ceriello A, Colagiuri S. International diabetes federation guideline for management of postmeal glucose: a review of recommendations. Diabet Med 2008;25:1151-6.

11 McGuire H, Longson D, Adler A, et al. Management of type 2 diabetes in adults: summary of updated NICE guidance. BMJ 2016;353:11575-4.

12 Miller KM, Beck RW, Bergenstal RM, et al. Evidence of a strong association between frequency of self-monitoring of blood 
glucose and hemoglobin A1c levels in T1D exchange clinic registry participants. Diabetes Care 2013;36:2009-14.

13 Vitale RJ, Pillai PB, Krishnan G, et al. The two levels of care for diabetes in a developing country: mechanisms for improved intermediate health outcomes. Diabetes Metab Syndr 2016;10:S90-4.

14 Claude Mbanya J, Aschner P, Chan JCN, et al. Self-monitoring of blood glucose (SMBG) and glycaemic control in Cameroon: results of the International Diabetes Management Practices Study (IDMPS). Diabetes Res Clin Pract 2017;126:198-201.

15 Ogle GD, Kim H, Middlehurst AC, et al. Financial costs for families of children with type 1 diabetes in lower-income countries. Diabet Med 2016;33:820-6.

16 Stephani V, Opoku D, Beran D. Self-Management of diabetes in sub-Saharan Africa: a systematic review. BMC Public Health 2018;18:1-11.

17 Park PH, Pastakia SD. Access to hemoglobin A1c in rural Africa: a difficult reality with severe consequences. J Diabetes Res 2018;2018:1:1-5.

18 National Institute of Statistics of Rwanda (NISR), Ministry of Finance and Economic Planning (MINECOFIN). Fourth Rwanda population and housing census. final results: main indicators report. Available: http://www.statistics.gov.rw/publication/rphc4-main-indicatorsreport (accessed 24 Aug 2018).

19 Tapela N. Diabetes in rural Rwanda: high retention and positive outcomes after 24 months of follow-up in the setting of chronic care integration. Int J Diabetes Clin Res 2016;3.
20 Ndayisaba A, Harerimana E, Borg R, et al. A clinical mentorship and quality improvement program to support health center nurses manage type 2 diabetes in rural Rwanda. J Diabetes Res 2017;2017:1-10.

21 Bukhman G, Kidder A. PIH guide to chronic care integration for endemic non-communicable diseases: Rwanda edition. Patners in Health Boston Massachusetts, 2011.

22 Eberly LA, Rusingiza E, Park PH, et al. Understanding the etiology of heart failure among the rural poor in sub-Saharan Africa: a 10-year experience from district hospitals in Rwanda. J Card Fail 2018;24:849-53.

23 Habineza H, Mutumbira C, Hedt-Gauthier BL, et al. Treating persistent asthma in rural Rwanda: characteristics, management and 24-month outcomes. Int J Tuberc Lung Dis 2017;21:1176-82.

24 Eberly LA, Rusangwa C, Ng'ang'a L, et al. Cost of integrated chronic care for severe non-communicable diseases at district hospitals in rural Rwanda. BMJ Glob Health 2019;4:e001449.

25 Kwan GF, Bukhman AK, Miller AC, et al. A simplified echocardiographic strategy for heart failure diagnosis and management within an integrated noncommunicable disease clinic at district hospital level for sub-Saharan Africa. JACC Heart Fail 2013;1:230-6.

26 Wambui Charity K, Kumar AMV, Hinderaker SG, et al. Do diabetes mellitus patients adhere to self-monitoring of blood glucose (SMBG) and is this associated with glycemic control? experiences from a SMBG program in Western Kenya. Diabetes Res Clin Pract 2016;112:37-43. 